\title{
Article \\ Clinical Outcomes of Pencil Beam Scanning Proton Therapy in Locally Advanced Non-Small Cell Lung Cancer: Propensity Score Analysis
}

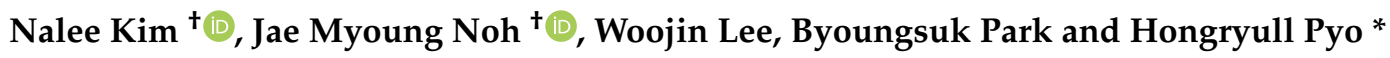 \\ Department of Radiation Oncology, Samsung Medical Center, Sungkyunkwan University School of Medicine, \\ Seoul 06351, Korea; nalee.kim@samsung.com (N.K.); jm2.noh@samsung.com (J.M.N.); \\ wj0616.lee@samsung.com (W.L.); bs1002.park@samsung.com (B.P.) \\ * Correspondence: hr.pyo@samsung.com \\ + These authors contributed equally to this work.
}

check for

updates

Citation: Kim, N.; Noh, J.M.; Lee, W.; Park, B.; Pyo, H. Clinical Outcomes of Pencil Beam Scanning Proton Therapy in Locally Advanced Non-Small Cell Lung Cancer: Propensity Score Analysis. Cancers 2021, 13, 3497. https://doi.org/ $10.3390 /$ cancers 13143497

Academic Editor: Hitoshi Ishikawa

Received: 8 June 2021

Accepted: 12 July 2021

Published: 13 July 2021

Publisher's Note: MDPI stays neutral with regard to jurisdictional claims in published maps and institutional affiliations.

Copyright: (c) 2021 by the authors. Licensee MDPI, Basel, Switzerland. This article is an open access article distributed under the terms and conditions of the Creative Commons Attribution (CC BY) license (https:// creativecommons.org/licenses/by/ $4.0 /)$.
Simple Summary: We analyzed the oncologic outcomes and toxicities after intensity-modulated radiation therapy (IMRT) or pencil beam scanning proton therapy (PBSPT) in patients with locally advanced non-small cell lung cancer treated with concurrent chemoradiation therapy. Due to an imbalance in baseline characteristics between IMRT and PBSPT, we used propensity score-based statistical analysis. Regarding radiation therapy planning, PBSPT exhibited superior sparing of the lung, heart, and spinal cord compared to intensity-modulated (photon) radiotherapy in patients with advanced NSCLC. However, PBSPT resulted in higher incidence of grade 3 or more dermatitis and esophagitis compared to IMRT. Despite declined baseline lung function, PBSPT demonstrated a comparable rate of symptomatic radiation pneumonitis compared to IMRT. PBSPT could be an effective and safe treatment technique with comparable locoregional control.

Abstract: This study compared the efficacy and safety of pencil beam scanning proton therapy (PBSPT) versus intensity-modulated (photon) radiotherapy (IMRT) in patients with stage III nonsmall cell lung cancer (NSCLC). We retrospectively reviewed 219 patients with stage III NSCLC who received definitive concurrent chemoradiotherapy between November 2016 and December 2018. Twenty-five patients (11.4\%) underwent PBSPT (23 with single-field optimization) and 194 patients (88.6\%) underwent IMRT. Rates of locoregional control (LRC), overall survival, and acute/late toxicities were compared between the groups using propensity score-adjusted analyses. Patients treated with PBSPT were older (median: 67 vs. 62 years) and had worse pulmonary function at baseline (both FEV1 and DLCO) compared to those treated with IMRT. With comparable target coverage, PBSPT exhibited superior sparing of the lung, heart, and spinal cord to radiation exposure compared to IMRT. At a median follow-up of 21.7 (interquartile range: 16.8-26.8) months, the 2-year LRC rates were $72.1 \%$ and $84.1 \%$ in the IMRT and PBSPT groups, respectively $(p=0.287)$. The rates of grade $\geq 3$ esophagitis were $8.2 \%$ and $20.0 \%$ after IMRT and PBSPT ( $p=0.073)$, respectively, while corresponding rates of grade $\geq 2$ radiation pneumonitis were $28.9 \%$ and $16.0 \%$, respectively $(p=0.263)$. PBSPT appears to be an effective and safe treatment technique even for patients with poor lung function, and it does not jeopardize LRC.

Keywords: proton therapy; pencil beam scanning; intensity-modulated radiotherapy; non-small cell lung cancer; radiation therapy; chemoradiation

\section{Introduction}

The mainstay treatment for locally advanced non-small cell lung cancer (NSCLC) is concurrent chemoradiation therapy (CCRT), with a median survival of 29 months [1]. Higher radiation therapy (RT) dose to improve locoregional control (LRC) [2] is often limited given the close proximity to the target volume of critical normal organs. 
Besides local control, radiation-related toxicities during RT could reduce patients' quality of life and treatment compliance, and some toxicities may be lethal [3]. Beginning typically at the second or third week of CCRT, acute RT-induced esophagitis occurred in $4-18 \%$ of patients, interfering with appropriate nutritional support and inducing longlasting dysphagia $[4,5]$. After several months, some patients experienced symptomatic radiation pneumonitis (RP), which may affect both quality of life and survival [6]. Owing to promising results from systemic treatments, concerns regarding late-onset toxicity (i.e., cardiotoxicity) have also recently increased [6,7]. Therefore, meticulous RT planning is needed to maximize the therapeutic ratio.

Given the physical advantages over photon RT, proton beam therapy (PBT) is expected to increase the therapeutic ratio by delivering a higher dose to the tumor and sparing normal organs. Along with early dosimetric studies [8,9], both retrospective and prospective clinical studies have reported promising results regarding efficacy and safety [10-14]. However, a recent randomized trial comparing PBT with passive scattering to intensity-modulated radiation therapy (IMRT) demonstrated that PBT did not confer a clinical benefit of RP or LRC over IMRT [15]. Nevertheless, technical advances in proton planning for intensity modulation as well as a change in the delivery form from wobbling to scanning are expected to provide further improvement in normal organ sparing and ultimate dose escalation of tumor [16]. Although several early reports of pencil beam scanning proton therapy (PBSPT) showed promising results, these were mainly focused on planning results. There is no randomized trial comparing PBSPT and IMRT in locally advanced NSCLC [17-21].

Herein, we retrospectively reviewed patients with stage III NSCLC treated with CCRT using PBSPT and compared them with those treated with CCRT using IMRT in terms of planning and clinical outcomes.

\section{Materials and Methods}

\subsection{Patient Population}

After approval from the institutional review board of Samsung Medical Center (No. 2020-01-034), we identified 283 patients with locally advanced NSCLC treated with CCRT between November 2016 and December 2018. Patients were excluded if they underwent PBSPT in combination with IMRT ( $n=12)$, did not complete RT $(n=13)$, and follow-up details were missing $(n=13)$. Ultimately, we retrospectively reviewed the medical records of 219 patients; 194 patients were treated with IMRT (IMRT group) and 25 patients were treated with PBSPT (PBSPT group), respectively. Informed consent was waived due to the retrospective nature of this study, and the study was performed in accordance with the provisions of the Declaration of Helsinki and Good Clinical Practice guidelines.

Using the Vmax 22 system (SensorMedics, Yorba Linda, CA, USA), spirometric analysis and diffusing capacity of the lungs for carbon monoxide (DLCO) values were assessed according to the American Thoracic Society/European Respiratory Society criteria. After obtaining absolute values of forced expiratory volume in one second (FEV1) and DLCO, the percentage of the predicted values for FEV1 and DLCO was calculated based on a representative Korean population [22]. Moderately low FEV1 and DLCO were defined as $50 \% \leq \mathrm{FEV} 1<70 \%$ predicted and $40 \% \leq \mathrm{DLCO}<60 \%$ predicted; severely low FEV1 and DLCO were defined as FEV1 $<50 \%$ and DLCO $<40 \%$ predicted [23].

\subsection{Radiation Therapy}

Based on all available clinical information, the gross tumor volume (GTV) was delineated in the average-intensity projection images reconstructed from 10 breathingphase, four-dimensional computed tomography (CT) scans. All patients underwent 18Ffluorodeoxyglucose positron emission tomography/computed tomography for determining GTV and detecting distant metastasis at the time of diagnosis. Internal target volume was established by expanding the GTV to include GTV for each phase of the breathing cycle. The clinical target volume (CTV) was generated by extending a $5 \mathrm{~mm}$ margin from 
the GTV. We routinely did not perform elective node irradiation in the uninvolved lymph node region. For planning target volume (PTV), a uniform $5 \mathrm{~mm}$ margin was placed on the CTV to account for setup uncertainty. Median total dose of $66 \mathrm{~Gy}$ (range, 59.4-74.0) with a fractional dose of 2.2 Gy (range, 2-2.2) was prescribed for PTV. Specifically, 66 Gy in 30 fractions was the most frequently adopted dose schedule in 152 patients (69.4\%) followed by 66 Gy in 33 fractions $(n=34,15.5 \%), 70$ Gy in 35 fractions $(n=13,5.9 \%), 60$ Gy in 30 fractions $(n=9,4.1 \%), 70.4 \mathrm{~Gy}$ in 32 fractions $(n=4,1.8 \%), 70 \mathrm{~Gy}$ in 35 fractions $(n=4,1.8 \%)$, and 74 Gy in 37 fractions $(n=3,1.4 \%)$. For all patients, $97 \%$ of the prescribed dose should encompass at least $95 \%$ of the CTV. The planning requirements for organ-at-risk were as follows: both lungs $\mathrm{V}_{5 \mathrm{GyE}}<65 \%$ (where $\mathrm{V}_{\mathrm{XXGyE}}$ is defined as the percentage of the volume receiving more than $\mathrm{XX}$ GyE), $\mathrm{V}_{10 \mathrm{GyE}}<45 \%, \mathrm{~V}_{20 \mathrm{GyE}}<35 \%$, mean lung dose $<20 \mathrm{GyE}$, heart $\mathrm{V}_{40 \mathrm{GyE}}<50 \%$, esophagus maximum dose $\left(\mathrm{D}_{\max }\right)<66 \mathrm{GyE}, \mathrm{V}_{45 \mathrm{GyE}}<50 \%$, and spinal cord $\mathrm{D}_{\max }<45 \mathrm{GyE}$.

Treatment planning for IMRT and PBSPT were generated on the Pinnacle treatment planning system, version 9.2 (Royal Phillips Electronics, Miami, FL, USA), and the RayStation (RaySearch Laboratories, Stockholm, Sweden), respectively. For IMRT, volumetric arc-modulated therapy was mostly adopted $(n=119,61.3 \%)$, followed by step-and-shoot method with 6 median coplanar beams (range $6-10)$ of $6 \mathrm{MV}$ photons $(n=71,36.6 \%)$, and Tomotherapy ( $n=4,2.1 \%$, Hi-Art TomoTherapy; Accuray, Madison, WI, USA). Regarding volumetric arc-modulated therapy, partial arc angle was used to minimize the radiation exposure of normal lung: $20-280^{\circ}( \pm 10) / 240-180^{\circ}( \pm 10)$ and $340-80^{\circ}( \pm 10) / 120-180^{\circ}$ $( \pm 10)$ for right and left lung cancer, respectively.

The relative biological effectiveness (RBE) for PBSPT was considered as a fixed value of 1.1. Most PBSPT plans were calculated under pencil beam algorithm $(n=21,84.0 \%)$ followed by Monte Carlo algorithm $(n=4,16.0 \%)$. Pencil beam algorithm, considering patients as a stack of semi-infinite layers, models the treatment beam with a summation of narrow pencil beam which interacts with medium for delivering energy [16]. In addition, a single-field optimization $(n=23,92.0 \%)$ with 2 fields $(n=18,72.0 \%)$ rather than 3 fields was utilized. All fields were delivered in the same day. For all patients in the PBSPT group, the continuous line-scanning method was used; detailed information on beam delivery and treatment procedure have been described previously [24]. Briefly, all PBSPT plans were robustness-optimized plans using minimax optimization [25]. Setup and range uncertainty was addressed as $5 \mathrm{~mm}$ and $+/-3.5 \%$, respectively.

Daily image guidance was performed with kilovoltage or megavoltage cone beam CT for IMRT and orthogonal kilovoltage X-ray images/or cone beam CT provided by VeriSuite (MedCom, Darmstadt, Germany) before each treatment session.

For additional dosimetric comparisons, matched IMRT plans were generated for the corresponding 25 patients in the PBSPT group. Matched IMRT plans were calculated with volumetric arc-modulated therapy and generated under the condition of achieving acceptable target coverage.

\subsection{Chemotherapy}

Overall, 206 patients (94.1\%) were treated with the paclitaxel/cisplatin regimen, 8 with paclitaxel/carboplatin, 4 with etoposide/cisplatin, and 1 with cisplatin alone. The paclitaxel/cisplatin or carboplatin regimen consisted of 6 cycles of weekly intravenous paclitaxel $\left(50 \mathrm{mg} / \mathrm{m}^{2}\right)$ with cisplatin $\left(25 \mathrm{mg} / \mathrm{m}^{2}\right)$ or carboplatin (area under curve of 1.5$)$. The first dose of chemotherapy was delivered on the first day of RT, and additional consolidation chemotherapy was performed following CCRT. There were 20 patients without epidermal growth factor receptor mutation who received consolidative durvalumab (monoclonal PD-L1 antibody) after CCRT: 1 and 19 patients in the PBSPT and IMRT group, respectively.

\subsection{Surveillance}

Once the planned treatment was completed, patients underwent chest $\mathrm{CT}$, pulmonary function test (PFT), and/or positron emission tomography/CT scan at 1 month after the 
planned CCRT, as well as every 3 months for the first 3 years and every 6 months thereafter. Local failure was defined as recurrence within the PTV; recurrent regional nodes outside the PTV were considered as regional failures. Recurrences beyond the primary and regional sites were denoted as distant failures. The acute and late toxicity events noted during and after RT were assessed by the treating physicians based on the Common Terminology Criteria for Adverse Events (CTCAE, ver 5.00). Absolute changes in PFT were calculated based on pre-treatment PFT values for available patients. Major cardiac adverse events were defined based on AHA/ACC guidelines: cardiac death, acute myocardial infarction, unstable angina hospitalization, and heart failure [26].

\subsection{Statistical Analysis}

Differences in continuous variables between the two groups were analyzed with Student's t-test (normally distributed data) and Mann-Whitney U test (non-normally distributed data). The Chi-square test or Fisher's exact test was used to evaluate differences in categorical variables between the two groups. The Wilcoxon signed rank test for nonparametric paired data was used to compare the PBSPT and paired IMRT plans. All events (including loco-regional failure and death) were measured from the day of CCRT to the time of the event. The Kaplan-Meier analysis was used to estimate LRC and OS. Multivariable analyses of LRC and OS were performed using Cox regression analysis; logistic regression analysis was used to identify the prognostic factors for grade $\geq 3$ esophagitis and grade $\geq 2$ RP. Factors with $p<0.10$ in univariable analysis were further assessed in multivariable analysis. Propensity scores were calculated using a multivariate logistic regression model, including sex (female vs. male), age (continuous), pathology (adenocarcinoma vs. non-adenocarcinoma), T stage (T1-2 vs. T3-4), N stage (N2 vs. N3), predicted value of FEV1 (continuous), predicted value of DLco (continuous), and PTV (continuous). Each patient was then assigned an estimated propensity score based on the patient's baseline characteristics. First, patients were matched using 1:2 optimal matching with a caliper distance set at 0.05 standard deviations of the logit of the propensity scores. Second, stabilized inverse probability of treatment weighting (IPTW) was used to adjust for any covariable imbalance. The standardized mean difference was used to evaluate the balance of covariate distribution between the 2 groups. A two-tailed $p<0.05$ was considered statistically significant. All statistical analyses were performed using IBM SPSS Statistics version 25 (IBM Corp., Armonk, NY, USA) and R (version 3.6.3; R Foundation for Statistical Computing, Vienna, Austria).

\section{Results}

\subsection{Baseline Characteristics}

In the studied patient population, the median age of the patients was 62 (interquartile range, 57-68) years, and most patients (97.7\%) had a good performance status of ECOG PS 0-1 (Table 1). Patients in the PBSPT group were older (median, 67 vs. $62, p=0.003$ ) and had less frequent contralateral mediastinal lymph node involvement (20.0\% vs. $43.3 \%$, $p=0.044)$ than those in the IMRT group. The median FEV1 (percentage predicted) and DLCO (percentage predicted) values in the PBSPT group were significantly lower than those in the IMRT group (both $p<0.05$, Figure S1). In addition, the prevalence of severely low FEV1 and moderately to severely low DLCO in the PBSPT group $(20.0 \%$ and $40.0 \%$, respectively) was higher than in the IMRT group ( $4.6 \%$ and $15.5 \%$, respectively). 
Table 1. Patient, tumor, and treatment characteristics.

\begin{tabular}{|c|c|c|c|c|c|}
\hline \multicolumn{2}{|c|}{ Variables } & \multirow{2}{*}{$\begin{array}{c}\text { IMRT } \\
n=194 \\
150(77.3)\end{array}$} & \multirow{2}{*}{$\begin{array}{c}\text { PBSPT } \\
n=25 \\
21(84.0)\end{array}$} & \multirow{2}{*}{$\begin{array}{l}\text { SMD } \\
0.170\end{array}$} & \multirow{2}{*}{$\frac{p \text {-Value }}{0.615}$} \\
\hline Sex & Male & & & & \\
\hline & Female & $44(22.7)$ & $4(16.0)$ & & \\
\hline Age, years & & $62.0[56.0 ; 67.0]$ & $67.0[61.0 ; 75.0]$ & 0.685 & 0.003 \\
\hline ECOG & $0-1$ & $191(98.5)$ & $23(92.0)$ & 0.306 & 0.101 \\
\hline \multirow[t]{2}{*}{ Smoking history } & Never smoker & $44(22.7)$ & $2(8.0)$ & 0.416 & 0.151 \\
\hline & Current or ex-smoker & $150(77.3)$ & $23(92.0)$ & & \\
\hline \multirow[t]{7}{*}{ Histology } & $\begin{array}{l}\text { Squamous cell } \\
\text { carcinoma }\end{array}$ & $75(38.7)$ & $15(60.0)$ & 0.468 & 0.067 \\
\hline & Adenocarcinoma & $114(58.8)$ & $9(36.0)$ & & \\
\hline & Etc. & $5(2.6)$ & $1(4.0)$ & & \\
\hline & NOS & 3 & 1 & & \\
\hline & LCNEC & 2 & & & \\
\hline & EGFR mutant, $n(\%)$ & $37(19.1)$ & $2(8.0)$ & 0.328 & 0.266 \\
\hline & ALK translocation, $n(\%)$ & $6(3.1)$ & $0(0.0)$ & 0.253 & 1.000 \\
\hline \multirow[t]{2}{*}{ Clinical T stage } & cT1-2 & $126(64.9)$ & $15(60.0)$ & 0.102 & 0.791 \\
\hline & cT3-4 & $68(35.1)$ & $10(40.0)$ & & \\
\hline \multirow[t]{2}{*}{ Clinical N stage } & $\mathrm{cN} 2$ & $72(26.8)$ & $13(52.0)$ & 0.534 & 0.018 \\
\hline & $\mathrm{cN} 3$ & $142(73.2)$ & $12(48.0)$ & & \\
\hline \multirow[t]{2}{*}{ N3 involvement region } & $\begin{array}{l}\text { Contralateral } \\
\text { mediastinum }\end{array}$ & $84(43.3)$ & $5(20.0)$ & 0.517 & 0.044 \\
\hline & Supraclavicular & $71(36.6)$ & $9(36.0)$ & 0.012 & 1.000 \\
\hline \multirow[t]{3}{*}{ Overall stage } & IIIA & $23(11.9)$ & $9(36.0)$ & 0.604 & 0.008 \\
\hline & IIIB & $138(71.1)$ & $12(48.0)$ & & \\
\hline & IIIC & $33(17.0)$ & $4(16.0)$ & & \\
\hline \multicolumn{6}{|c|}{ Pre-treatment pulmonary function test } \\
\hline \multicolumn{2}{|c|}{ FEV1, L } & $2.50(2.10 ; 3.06)$ & $2.35(1.61 ; 2.81)$ & 0.487 & 0.040 \\
\hline \multicolumn{2}{|c|}{ FEV1, \% } & $84.0(71.0 ; 95.0)$ & $72.0(57.0 ; 88.0)$ & 0.420 & 0.042 \\
\hline \multicolumn{2}{|c|}{ FEV1 < 70\% (moderate to severe) } & $44(22.7)$ & $10(40.0)$ & & 0.100 \\
\hline \multicolumn{2}{|c|}{ FEV1 < 50\% (severe) } & $9(4.6)$ & $5(20.0)$ & & 0.013 \\
\hline \multicolumn{2}{|c|}{ DLCO, $\%$} & $79.5(66.0 ; 92.0)$ & $65.0(51.0 ; 79.0)$ & 0.654 & 0.002 \\
\hline \multicolumn{2}{|c|}{ DLCO < 60\% (moderate to severe) } & $30(15.5)$ & $10(40.0)$ & & 0.006 \\
\hline \multicolumn{2}{|c|}{ DLCO $<40 \%$ (severe) } & $2(1.0)$ & $1(4.0)$ & & 0.306 \\
\hline
\end{tabular}

Values are presented as number of patients (\%) or median (interquartile range). Abbreviations: IMRT, intensity-modulated radiation therapy; PBSPT, pencil beam scanning proton therapy; SMD, standardized mean difference; SD, standard deviation; IQR, interquartile range; NOS, not otherwise specified; LCNEC, large cell neuroendocrine carcinoma; EGFR, epidermal growth factor receptor; ALK, anaplastic lymphoma kinase; FEV1, forced expiratory volume in $1 \mathrm{~s}$; DLCO, diffusing capacity of the lung for carbon monoxide.

\subsection{Radiation Therapy Characteristics}

There was no significant difference in total prescription dose and target volumes between the two groups (Figure 1, Table S1). Although PBSPT plans covered $95 \%$ of PTV with a lower dose than IMRT plans $(94.8 \%$ vs. $97.1 \%, p=0.013)$, both plans encompassed $100 \%$ of CTV under the acceptable institutional criteria, presenting $96.2 \%$ and $96.7 \%$ of the prescribed dose, respectively $(p=0.314)$. Regarding both lungs, PBSPT significantly reduced not only the average dose but also $\mathrm{V}_{5 \mathrm{GyE}}, \mathrm{V}_{10 \mathrm{GyE}}$, and $\mathrm{V}_{20 \mathrm{GyE}}($ all $p<0.001$ ). Although Dmax of esophagus in the IMRT group was higher than that in the PBSPT group (71.2 vs. $69.7 \mathrm{GyE}, p=0.042), \mathrm{V}_{45 \mathrm{GyE}}, \mathrm{V}_{55 \mathrm{GyE}}$, and $\mathrm{V}_{66 \mathrm{GyE}}$ were comparable between the two groups (all $p>0.05$ ). Plans in the PBSPT group also showed lower mean heart dose (7.7 vs. $12.8 \mathrm{GyE}, p=0.006$ ) and $\mathrm{D}_{\max }$ of spinal cord (31.0 vs. $\left.42.6 \mathrm{GyE}, p<0.001\right)$ than those in the IMRT group. 


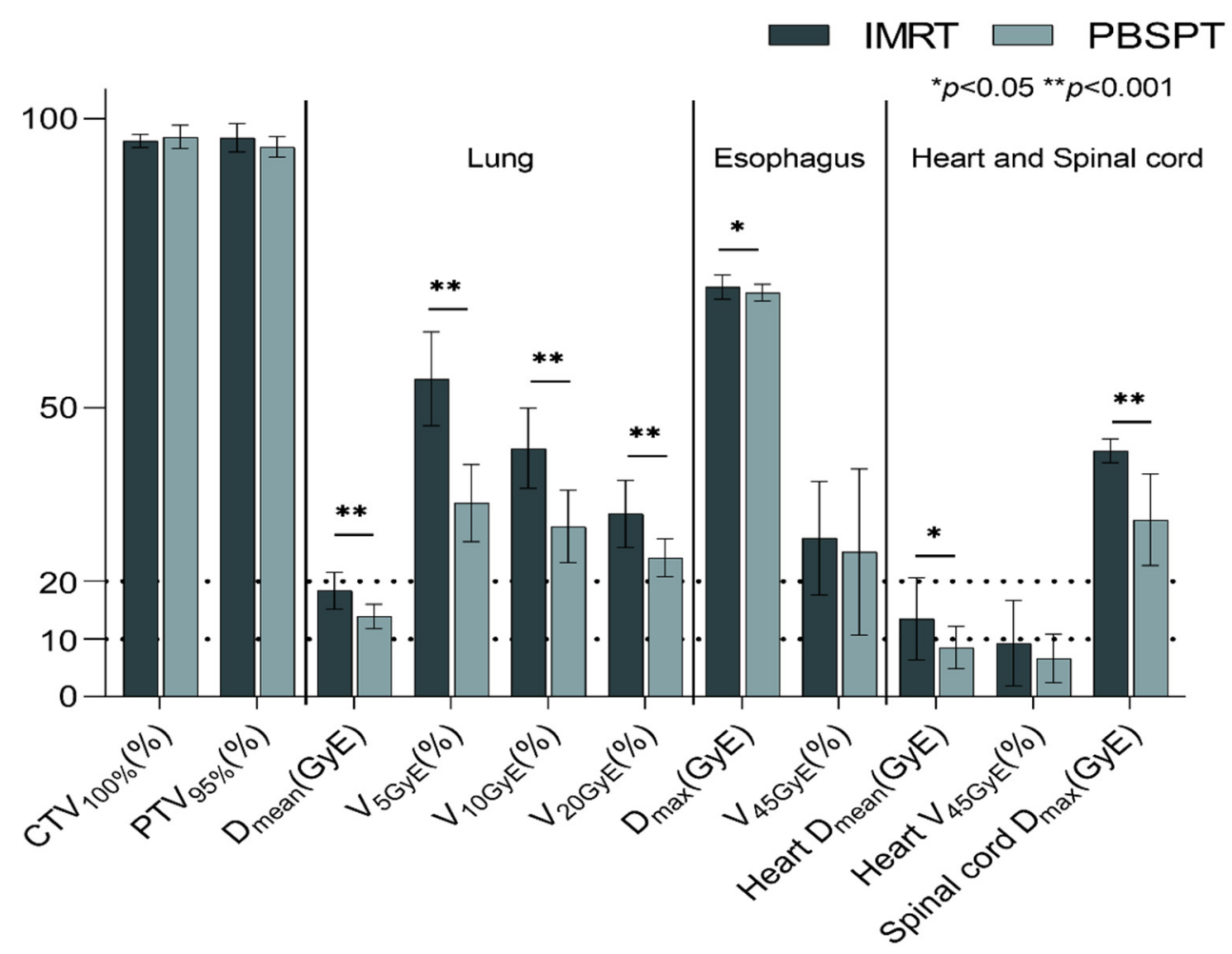

Figure 1. Dose-volume parameters for target volume and normal organs in patients treated with IMRT and PBSPT. Data are presented as the median, interquartile range. Abbreviations: IMRT, intensity-modulated radiation therapy; PBSPT, pencil beam scanning proton therapy; GyE, gray equivalent; CTV, clinical target volume; PTV, planning target volume; $\mathrm{V}_{\mathrm{XX} \%}$, volume receiving $X X \%$ of the prescription dose; $V_{X X G y E}$, volume receiving more than $X X$ Gy; $D_{\text {mean }}$, mean dose; $D_{\text {max }}$, maximum dose.

\subsection{Oncologic Outcomes}

With a median follow-up of 21.7 (interquartile range, 16.8-26.8) months for the entire cohort, the rates of 2-year LRC and OS were $72.8 \%$ and $82.9 \%$, respectively. During the last follow-up, 50 (22.8\%) and 117 patients (53.4\%) experienced locoregional failures and distant metastases. The rates of 2-year LRC were $72.1 \%$ and $84.1 \%$ in the IMRT and PBSPT groups, respectively ( $p=0.287$, Figure $2 \mathrm{~A}$ ). Patients in the PBSPT group showed lower OS rates than those in the IMRT group (rates of 2-year OS: $74.9 \%$ vs. $84.4 \%, p=0.061$, Figure 2B). Multivariable analysis revealed that treatment modality had little impact on both LRC and OS; only GTV $\geq 100$ cc showed borderline significance in LRC (HR 1.74, $p=0.069$, Table 2). 

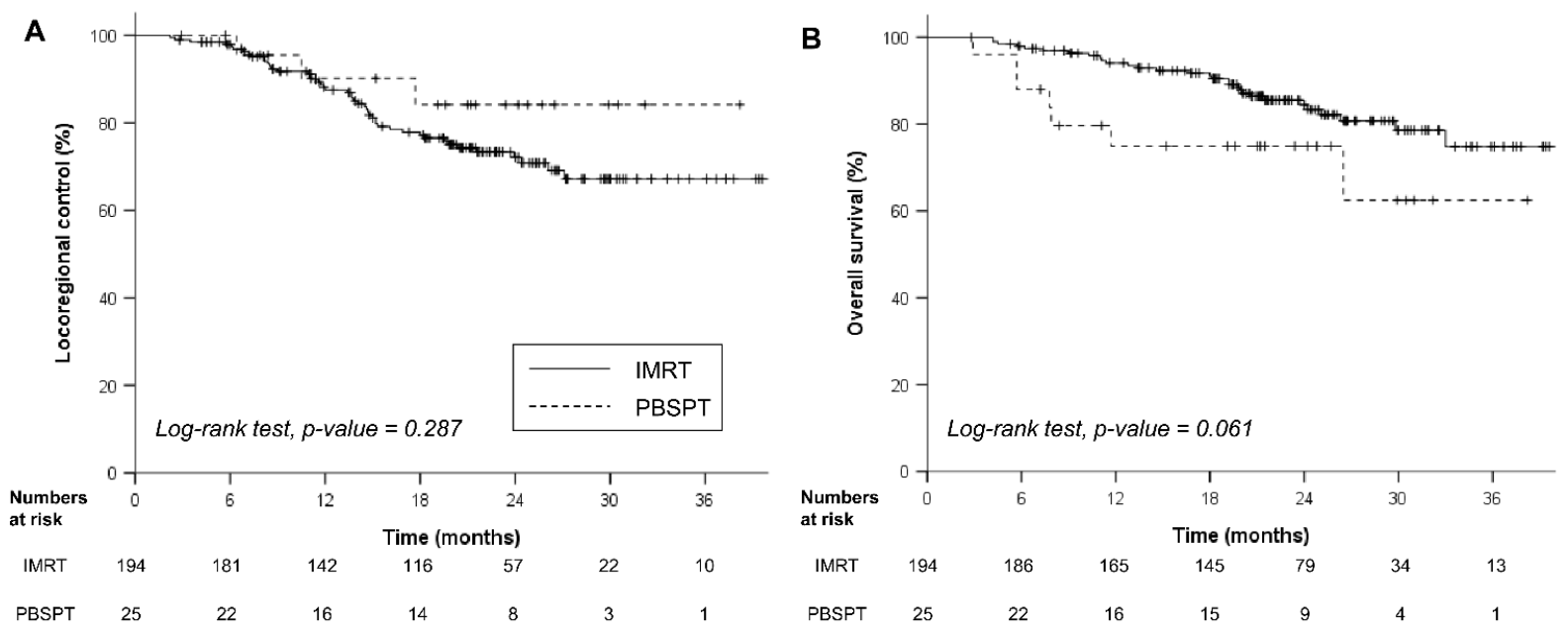

Figure 2. Clinical outcomes after IMRT and PBSPT: LRC (A) and OS (B). Abbreviations: IMRT, intensity-modulated radiation therapy; PBSPT, pencil beam scanning proton therapy; LRC, locoregional control; OS, overall survival.

Table 2. Prognostic factors for locoregional control and overall survival.

\begin{tabular}{|c|c|c|c|c|c|c|c|}
\hline \multicolumn{2}{|c|}{ Locoregional Control } & \multicolumn{3}{|c|}{ Univariable Analysis } & \multicolumn{3}{|c|}{ Multivariable Analysis } \\
\hline Variables & (Reference vs.) & HR & $(95 \% \mathrm{CI})$ & $p$-Value & & & \\
\hline RT modality & (IMRT vs. PBSPT) & 0.54 & $(0.17-1.72)$ & 0.296 & 0.43 & $(0.13-1.41)$ & 0.165 \\
\hline Sex & (female vs. male) & 1.80 & $(0.84-3.83)$ & 0.130 & & & \\
\hline Age & (<65 vs. $\geq 65$ years) & 0.68 & $(0.37-1.25)$ & 0.218 & & & \\
\hline Histology & (non-ADC vs. ADC) & 0.52 & $(0.30-0.92)$ & 0.024 & 0.58 & $(0.33-1.05)$ & 0.071 \\
\hline EGFR mutation & (wildtype vs. mutant) & 0.51 & $(0.22-1.20)$ & 0.122 & & & \\
\hline Clinical T stage & (T1-2 vs. T3-4) & 1.50 & $(0.85-2.63)$ & 0.162 & & & \\
\hline Clinical N stage & (N2 vs. N3) & 0.94 & $(0.50-1.78)$ & 0.858 & & & \\
\hline $\begin{array}{l}\text { Contralateral mediastinal } \\
\text { lymph node }\end{array}$ & (no vs. yes) & 1.20 & $(0.69-2.10)$ & 0.515 & & & \\
\hline SCF lymph node & (no vs. yes) & 0.81 & $(0.45-1.46)$ & 0.486 & & & \\
\hline GTV & (<100 vs. $\geq 100 \mathrm{cc})$ & 1.90 & $(1.06-3.38)$ & 0.030 & 1.74 & $(0.96-3.16)$ & 0.069 \\
\hline PTV & $(<550$ vs. $\geq 550$ cc $)$ & 1.18 & $(0.68-2.05)$ & 0.567 & & & \\
\hline Fractional RT dose & (2.0 vs. $2 . \overline{\mathrm{GyE}})$ & 0.21 & $(0.01-3.86)$ & 0.294 & & & \\
\hline Total RT dose & $(\leq 66$ vs. $>66$ GyE) & 0.97 & $(0.38-2.44)$ & 0.944 & & & \\
\hline $\mathrm{BED}_{10}$ & $(\leq 80$ vs. $>80 \mathrm{GyE})$ & 0.75 & $(0.41-1.38)$ & 0.353 & & & \\
\hline \multicolumn{2}{|c|}{ Overall survival } & \multicolumn{3}{|c|}{ Univariable } & \multicolumn{3}{|c|}{ Multivariable } \\
\hline Variables & (reference vs.) & HR & $(95 \% \mathrm{CI})$ & $p$-value & HR & $(95 \% \mathrm{CI})$ & $p$-value \\
\hline RT modality & (IMRT vs. PBSPT) & 2.17 & $(0.95-4.94)$ & 0.066 & 1.64 & $(0.70-3.81)$ & 0.254 \\
\hline Sex & (female vs. male) & 2.17 & $(0.95-4.94)$ & 0.294 & & & \\
\hline Age & (<65 vs. $\geq 65$ years) & 1.56 & $(0.68-3.58)$ & 0.030 & 1.66 & $(0.82-3.36)$ & 0.156 \\
\hline Histology & (non-ADC vs. ADC) & 2.10 & $(1.08-4.10)$ & 0.006 & 0.54 & $(0.26-1.13)$ & 0.103 \\
\hline EGFR mutation & (wildtype vs. mutant) & 0.55 & $(0.16-1.17)$ & 0.098 & 0.66 & $(0.21-2.00)$ & 0.459 \\
\hline Clinical T stage & (T1-2 vs. T3-4) & 1.28 & $(0.66-2.49)$ & 0.470 & & & \\
\hline Clinical $\mathrm{N}$ stage & (N2 vs. N3) & 0.65 & $(0.33-1.31)$ & 0.230 & & & \\
\hline $\begin{array}{l}\text { Contralateral mediastinal } \\
\text { lymph node }\end{array}$ & (no vs. yes) & 0.85 & $(0.44-1.64)$ & 0.625 & & & \\
\hline SCF lymph node & (no vs. yes) & 0.98 & $(0.50-1.90)$ & 0.950 & & & \\
\hline FEV1 $(\%)$ & $(\geq 70$ vs. $<70 \%)$ & 0.85 & $(0.39-1.86)$ & 0.687 & & & \\
\hline DLCO $(\%)$ & $(\geq 60$ vs. $<60 \%)$ & 1.64 & $(0.75-3.61)$ & 0.216 & & & \\
\hline GTV & $(<100$ vs. $\geq 100 \mathrm{cc})$ & 1.48 & $(0.77-2.86)$ & 0.243 & & & \\
\hline Total RT dose & (>66 vs. $\leq 66 \mathrm{GyE})$ & 4.55 & $(0.63-33.3)$ & 0.132 & & & \\
\hline $\mathrm{BED}_{10}$ & $(>80$ vs. $\leq 80$ GyE) & 1.01 & $(0.48-2.12)$ & 0.986 & & & \\
\hline
\end{tabular}

The foreparts of the parentheses were set as the reference group. Abbreviations: HR, hazard ratio; $\mathrm{CI}$, confidence interval; RT, radiation therapy; IMRT, intensity-modulated radiation therapy; PBSPT, pencil beam scanning proton therapy; ADC, adenocarcinoma; EGFR, epidermal growth factor receptor; SCF, supraclavicular fossa; GTV, gross tumor volume; PTV, planning target volume; GyE, gray relative biologic effectiveness; $\mathrm{BED}_{10}$, biological effective dose with $\alpha / \beta$ of 10; FEV1, forced expiratory volume in $1 \mathrm{~s}$; DLCO, diffusing capacity of the lung for carbon monoxide. 


\subsection{Toxicity}

The toxicities reported in this study are summarized in Table 3. Twenty-four $(11.0 \%)$ grade 3 or more acute toxic events were observed in the entire cohort. Among 21 patients with grade 3 or more esophagitis, all patients were hospitalized with temporary total parenteral nutrition and five patients required tube feeding. There was a trend toward frequent grade $\geq 3$ esophagitis with PBSPT ( $20.0 \%$ vs. $8.2 \%, p=0.073$, Figure 3$)$; grade $\geq 3$ radiation dermatitis was more frequently observed in the PBSPT group than the IMRT group $(8.0 \%$ vs. $0.5 \%, p=0.035$ ). PBSPT was associated with frequent grade $\geq 3$ esophagitis after multivariable analysis (odds ratio (OR) 3.68, Table 4). Additionally, esophagus $\mathrm{V}_{45 \mathrm{GyE}} \geq 35 \%$ was also related to the incidence of grade $\geq 3$ esophagitis in multivariable analysis. There were two patients in the IMRT group who experienced trachea-esophageal fistula requiring surgical intervention. There were 60 patients $(27.4 \%)$ who experienced symptomatic RP with comparable incidence between the IMRT and PBSPT groups (28.9\% vs. $16.0 \%, p=0.263)$. Multivariable analysis showed that both-lung $\mathrm{V}_{10 \mathrm{GyE}} \geq 45 \%$ significantly increased the grade $\geq 2 \mathrm{RP}$ (OR 4.37, Table 4). Differences in declined pulmonary function between the IMRT and PBSPT groups were not statistically significant throughout the follow-up period (Figure S2). Regarding cardiac adverse events, there was no significant difference between the IMRT and PBSPT groups (9.3\% vs. $8.0 \%$, Table 4$)$.

Table 3. Detailed profiles of treatment-related adverse events *.

\begin{tabular}{|c|c|c|c|c|}
\hline Category & Grade & $\begin{array}{c}\text { IMRT } \\
n=194 \\
n(\%)\end{array}$ & $\begin{array}{c}\text { PBSPT } \\
n=25 \\
n(\%)\end{array}$ & $p$-Value \\
\hline \multicolumn{5}{|l|}{ Acute toxicity } \\
\hline \multirow[t]{5}{*}{ Esophagitis } & Grade 0 & $29(14.9)$ & $4(16.0)$ & 0.286 \\
\hline & Grade 1 & $69(35.6)$ & $9(36.0)$ & \\
\hline & Grade 2 & $80(41.2)$ & $7(28.0)$ & \\
\hline & Grade 3 & $15(7.7)$ & $5(20.0)$ & \\
\hline & Grade 4 & $1(0.5)$ & $0(0.0)$ & \\
\hline \multirow[t]{4}{*}{ Radiation dermatitis } & Grade 0 & $161(83.0)$ & $19(76.0)$ & 0.013 \\
\hline & Grade 1 & $17(8.8)$ & $0(0.0)$ & \\
\hline & Grade 2 & $15(7.7)$ & $4(16.0)$ & \\
\hline & Grade 3 & $1(0.5)$ & $2(8.0)$ & \\
\hline \multicolumn{5}{|l|}{ Late toxicity } \\
\hline \multirow[t]{4}{*}{ Radiation pneumonitis } & Grade 0 & 115 (59.3) & $18(72.0)$ & 0.648 \\
\hline & Grade 1 & $23(11.9)$ & $3(12.0)$ & \\
\hline & Grade 2 & $47(24.2)$ & $4(16.0)$ & \\
\hline & Grade 3 & $9(4.6)$ & $0(0.0)$ & \\
\hline \multirow[t]{4}{*}{ Late esophageal toxicity } & Grade 0 & $191(98.5)$ & $24(96.0)$ & 0.386 \\
\hline & Grade 1 & $0(0.0)$ & $0(0.0)$ & \\
\hline & Grade 2 & $1(0.5)$ & $1(4.0)$ & \\
\hline & Grade 3 & $2(1.0)$ & $0(0.0)$ & \\
\hline Major cardiac adverse events & & $18(9.3)$ & $2(8.0)$ & 1.000 \\
\hline
\end{tabular}

* Grades refers to grading systems of Common Terminology Criteria for Adverse Events. Abbreviations: IMRT, intensity-modulated radiation therapy (photon); PBSPT, pencil beam scanning proton therapy. 
Table 4. Prognostic factors for grade $\geq 3$ acute esophagitis and grade $\geq 2$ radiation pneumonitis.

\begin{tabular}{|c|c|c|c|c|c|c|c|}
\hline \multicolumn{2}{|c|}{ Grade $\geq 3$ Acute Esophagitis } & \multicolumn{3}{|c|}{ Univariable Analysis } & \multicolumn{3}{|c|}{ Multivariable Analysis } \\
\hline Variables & (Reference vs.) & OR & $(95 \% \mathrm{CI})$ & $p$-Value & OR & $(95 \% \mathrm{CI})$ & $p$-Value \\
\hline RT modality & (IMRT vs. PBSPT) & 2.78 & $(0.84-8.00)$ & 0.070 & 3.68 & $(0.97-12.88)$ & 0.045 \\
\hline Sex & (female vs. male) & 0.33 & $(0.13-0.85)$ & 0.019 & 0.32 & $(0.06-1.74)$ & 0.181 \\
\hline Age & (<65 vs. $\geq 65$ years) & 1.2 & $(0.47-2.98)$ & 0.698 & & & \\
\hline Smoking history & (never vs. ever) & 0.39 & $(0.15-1.04)$ & 0.049 & 0.74 & $(0.13-4.30)$ & 0.735 \\
\hline Clinical T stage & (T1-2 vs. T3-4) & 0.89 & $(0.33-2.26)$ & 0.818 & & & \\
\hline Clinical N stage & (N2 vs. N3) & 0.53 & $(0.21-1.35)$ & 0.170 & & & \\
\hline Contralateral & & & & & & & \\
\hline $\begin{array}{l}\text { mediastinal lymph } \\
\text { node }\end{array}$ & (no vs. yes) & 0.89 & $(0.34-2.21)$ & 0.803 & & & \\
\hline SCF lymph node & (no vs. yes) & 0.86 & $(0.31-2.16)$ & 0.749 & & & \\
\hline PTV & $(<550$ vs. $\geq 550$ cc $)$ & 1.39 & $(0.56-3.54)$ & 0.479 & & & \\
\hline Fractional RT dose & (2.0 vs. $2.2 \mathrm{GyE})$ & 2.37 & $(0.76-10.38)$ & 0.181 & & & \\
\hline Total RT dose & $(\leq 66$ vs. $>66$ GyE $)$ & 0.69 & $(0.27-1.66)$ & 0.408 & & & \\
\hline Esophagus $\mathrm{D}_{\max }$ & $(<70$ vs. $\geq 70$ GyE $)$ & 2.65 & $(0.94-9.46)$ & 0.090 & 2.46 & $(0.68-10.66)$ & 0.193 \\
\hline Esophagus $\mathrm{V}_{45 \mathrm{GyE}}$ & $(<35$ vs. $\geq 35 \%)$ & 4.33 & $(1.73-11.50)$ & 0.002 & 3.98 & $(1.12-19.02)$ & 0.049 \\
\hline Esophagus $\mathrm{V}_{55 \mathrm{GyE}}$ & $(<20$ vs. $\geq 20 \%)$ & 3.14 & $(1.18-9.89)$ & 0.032 & 0.76 & $(0.13-3.69)$ & 0.736 \\
\hline Esophagus $\mathrm{V}_{66 \mathrm{GyE}}$ & $(<10$ vs. $\geq 10 \%)$ & 2.05 & $(0.83-5.24)$ & 0.122 & & & \\
\hline \multicolumn{2}{|c|}{ Grade $\geq 2$ radiation pneumonitis } & \multicolumn{3}{|c|}{ Univariable analysis } & \multicolumn{3}{|c|}{ Multivariable analysis } \\
\hline Variables & (reference vs.) & OR & $(95 \% \mathrm{CI})$ & $p$-value & OR & $(95 \% \mathrm{CI})$ & $p$-value \\
\hline RT modality & (IMRT vs. PBSPT) & 0.47 & $(0.13-1.30)$ & 0.183 & 0.88 & $(0.24-2.64)$ & 0.837 \\
\hline Sex & (female vs. male) & 0.69 & $(0.35-1.41)$ & 0.298 & & & \\
\hline Age & (<65 vs. $\geq 65$ years) & 1.07 & $(0.58-1.96)$ & 0.825 & & & \\
\hline Smoking history & (never vs. ever) & 0.64 & $(0.32-1.30)$ & 0.208 & & & \\
\hline Clinical T stage & (T1-2 vs. T3-4) & 1.06 & $(0.57-1.96)$ & 0.842 & & & \\
\hline $\begin{array}{c}\text { Clinical N stage } \\
\text { Contralateral }\end{array}$ & (N2 vs. N3) & 0.64 & $(0.32-1.21)$ & 0.166 & & & \\
\hline $\begin{array}{l}\text { mediastinal lymph } \\
\text { node }\end{array}$ & (no vs. yes) & 0.72 & $(0.38-1.32)$ & 0.298 & & & \\
\hline SCF lymph node & (no vs. yes) & 1.01 & $(0.54-1.86)$ & 0.979 & & & \\
\hline FEV1 $(\%)$ & $(\geq 70$ vs. $<70 \%)$ & 0.80 & $(0.38-1.59)$ & 0.529 & & & \\
\hline DLCO $(\%)$ & $(\geq 60$ vs. $<60 \%)$ & 0.86 & $(0.38-1.84)$ & 0.707 & & & \\
\hline PTV & $(<550$ vs. $\geq 550$ cc $)$ & 1.61 & $(0.89-2.95)$ & 0.121 & & & \\
\hline Fractional RT dose & $(2.0$ vs. $2.2 \mathrm{GyE})$ & 1.15 & $(0.59-2.32)$ & 0.691 & & & \\
\hline Total RT dose & $(\leq 66$ vs. $>66$ GyE $)$ & 0.76 & $(0.24-2.03)$ & 0.605 & & & \\
\hline Both-lung $\mathrm{D}_{\text {mean }}$ & $(<20$ vs. $\geq 20$ GyE $)$ & 2.79 & $(1.51-5.19)$ & 0.001 & 1.71 & $(0.62-4.74)$ & 0.297 \\
\hline Both-lung $\mathrm{V}_{5 \mathrm{GyE}}$ & $(<65$ vs. $\geq 65 \%)$ & 1.86 & $(0.90-3.76)$ & 0.087 & 0.61 & $(0.24-1.51)$ & 0.292 \\
\hline Both-lung $\mathrm{V}_{10 \mathrm{GyE}}$ & $(<45$ vs. $\geq 45 \%)$ & 3.78 & $(2.04-7.10)$ & $<0.001$ & 4.37 & $(1.63-12.12)$ & 0.004 \\
\hline Both-lung $\mathrm{V}_{20 \mathrm{GyE}}$ & $(<35$ vs. $\geq 35 \%)$ & 2.60 & $(1.40-4.86)$ & 0.003 & 0.69 & $(0.20-2.23)$ & 0.542 \\
\hline
\end{tabular}

The foreparts of the parentheses were set as the reference group. Abbreviations: OR, odds ratio; CI, confidence interval; RT, radiation therapy; IMRT, intensity-modulated radiation therapy; PBSPT, pencil beam scanning proton therapy; SCF, supraclavicular fossa; FEV1, forced expiratory volume in $1 \mathrm{~s}$; DLCO, diffusing capacity of the lung for carbon monoxide; PTV, planning target volume; GyE, gray relative biologic effectiveness; BED10, biological effective dose with $\alpha / \beta$ of $10 ; \mathrm{V}_{\mathrm{XXGyE}}=$ volume receiving more than XX GyE. 


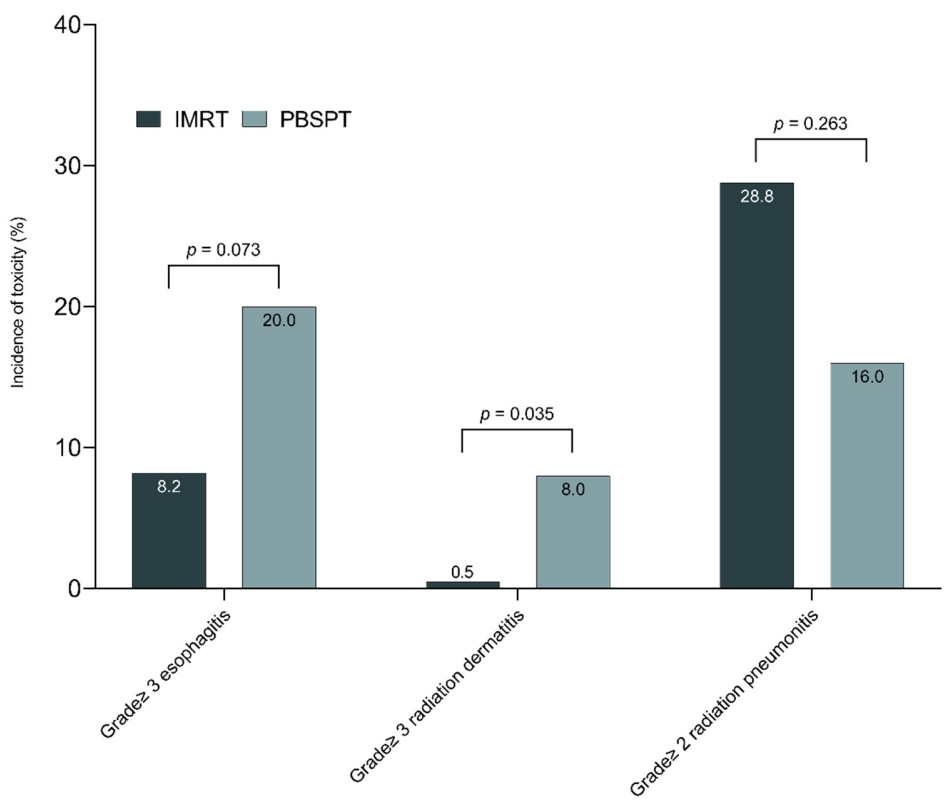

Figure 3. The incidence of specific treatment-related toxicities in patients treated with IMRT and PBSPT. Abbreviations: IMRT, intensity-modulated radiation therapy; PBSPT, pencil beam scanning proton therapy.

\subsection{Dosimetric Comparison for Matched IMRT and PBSPT Plans}

After propensity score matching, 50 patients from the IMRT group and 25 patients from the PBSPT group were included following analysis with well-balanced baseline characteristics. In addition, baseline characteristics, except for T stage, were adequately balanced after IPTW (Table 5).

Table 5. Patient, tumor, and treatment characteristics after propensity score matching and weighting.

\begin{tabular}{|c|c|c|c|c|c|c|c|}
\hline & & \multicolumn{3}{|c|}{ Propensity Score Matching } & \multicolumn{3}{|c|}{ IPTW } \\
\hline \multicolumn{2}{|c|}{ Variables } & IMRT & PBSPT & IM & IMRT & PBSPT & \\
\hline Sex & \multirow[t]{2}{*}{ Male } & $42(84.0)$ & $21(84.0)$ & $<0.001$ & $153.7(78.7)$ & $36.2(67.5)$ & 0.238 \\
\hline Age, years & & $67.7(7.0)$ & $67.5(8.8)$ & 0.033 & $62.0(9.0)$ & $60.1(8.8)$ & 0.217 \\
\hline ECOG & $0-1$ & $50(100.0)$ & $23(92.0)$ & 0.417 & 194 (98.5) & $52.5(97.9)$ & 0.039 \\
\hline \multirow[t]{2}{*}{ Histology } & $\mathrm{ADC}$ & $18(36.0)$ & $9(36.0)$ & $<0.001$ & $110.0(56.4)$ & $35.3(65.9)$ & 0.196 \\
\hline & EGFR mutant & $5(10.00)$ & $2(8.0)$ & 0.070 & $35.3(17.9)$ & $14.9(27.8)$ & 0.238 \\
\hline $\begin{array}{l}\text { Clinical T } \\
\text { stage }\end{array}$ & cТ3-4 & $19(38.0)$ & $10(40.0)$ & 0.121 & $71.6(36.4)$ & $33.8(63.2)$ & 0.557 \\
\hline $\begin{array}{l}\text { Clinical N } \\
\text { stage }\end{array}$ & $\mathrm{cN3}$ & $26(52.0)$ & $12(48.0)$ & 0.080 & $138.7(70.4)$ & $37.3(69.6)$ & 0.017 \\
\hline \multirow[t]{2}{*}{ N3 region } & $\begin{array}{l}\text { Contralateral } \\
\text { mediastinum }\end{array}$ & $13(26.0)$ & $5(20.0)$ & 0.143 & $82.4(41.8)$ & $20.2(37.7)$ & 0.084 \\
\hline & Supraclavicular & $14(28.0)$ & $9(36.0)$ & 0.172 & $68.7(34.9)$ & $24.1(45.0)$ & 0.207 \\
\hline \multicolumn{8}{|c|}{ Pre-treatment pulmonary function test } \\
\hline \multirow{2}{*}{\multicolumn{2}{|c|}{$\begin{array}{l}\text { FEV1, \% } \\
\text { DLCO, \% }\end{array}$}} & $74.4(20.0)$ & $73.1(24.2)$ & 0.061 & $81.1(18.7)$ & $78.6(21.7)$ & 0.112 \\
\hline & & $71.5(22.0)$ & $67.0(19.3)$ & 0.216 & $78.0(19.2)$ & $82.3(19.4)$ & 0.121 \\
\hline \multicolumn{8}{|c|}{ Radiation therapy } \\
\hline \multirow{3}{*}{\multicolumn{2}{|c|}{$\begin{array}{l}\text { Gross tumor volume, cc } \\
\text { Clinical target volume, cc } \\
\text { Planning target volume, cc }\end{array}$}} & $191.8(188.8)$ & $178.0(151.2)$ & 0.080 & $156.9(162.8)$ & $176.5(133.6)$ & 0.131 \\
\hline & & $443.4(307.4)$ & $453.6(307.3)$ & 0.033 & $378.9(271.6)$ & $440.3(260.8)$ & 0.231 \\
\hline & & $704.8(405.3)$ & $734.4(437.9)$ & 0.070 & $628.0(361.1)$ & $711.7(380.8)$ & 0.226 \\
\hline
\end{tabular}

Values are presented as number of patients (\%) or mean (standard deviation). Abbreviations: IPTW, inverse probability of treatment weighting; IMRT, intensity-modulated radiation therapy; PBSPT, pencil beam scanning proton therapy; SMD, standardized mean difference; ADC, adenocarcinoma; EGFR, Epidermal growth factor receptor; FEV1, forced expiratory volume in $1 \mathrm{~s}$; DLCO, diffusing capacity of the lung for carbon monoxide. 
A dose-volume histogram for an average of matched IMRT and PBSPT plans is shown in Figure 4. Although both plans achieved similar CTV/PTV coverage under the institutional dose constraints, PBSPT significantly reduced the volume of lungs, heart, and spinal cord exposed to low-to-high doses of radiation (Table S2). On the contrary, PBSPT increased the intermediate-to-high doses delivered to the esophagus (45, 55, and $66 \mathrm{GyE})$, whereas PBSPT delivered similar maximum doses to the esophagus (69.7 vs. $71.0 \mathrm{GyE}$, $p=0.241)$ when compared to matched IMRT plans.
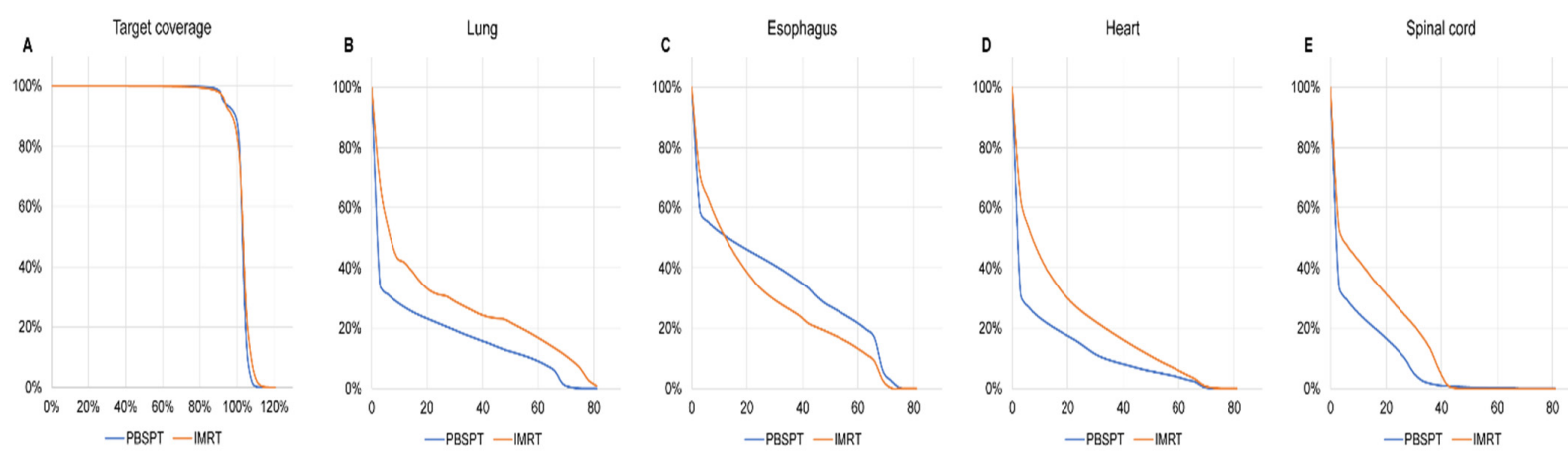

Figure 4. Dose-volume histogram for an average of matched intensity-modulated radiation therapy (IMRT) and pencil beam scanning proton therapy (PBSPT) plans: planning target volume (A); both lungs (B); esophagus (C); heart (D); spinal cord (E). Abbreviations: IMRT, intensity-modulated radiation therapy; PBSPT, pencil beam scanning proton therapy.

\subsection{Oncologic and Toxicity Outcomes for Propensity Score-Adjusted Patients}

After PSM and IPTW, PBSPT showed comparable LRC and OS outcomes (Figure 5, Table 6). Regarding toxicity, PBSPT was associated with frequent grade $\geq 3$ esophagitis after IPTW (OR 5.33, Table 6). In addition, PBSPT showed a borderline benefit over IMRT for grade 2 or more RP in propensity score-adjusted analyses (Table 6).
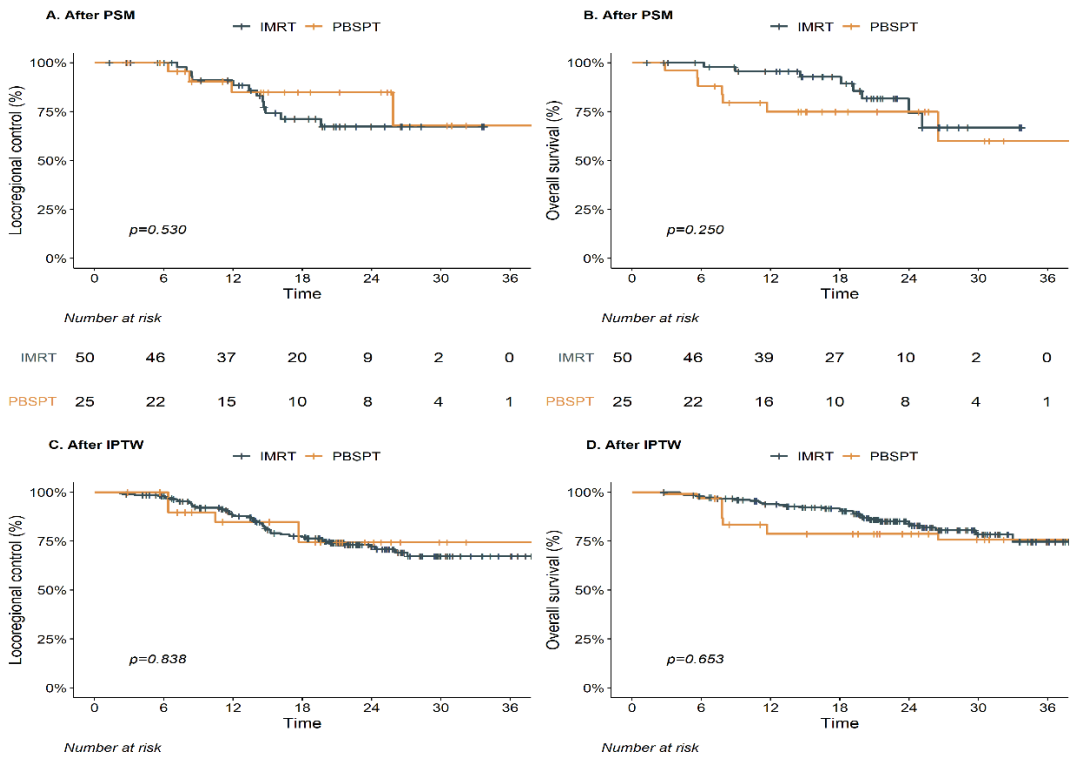

$\begin{array}{llllllllllllllll}\text { IMRT } & 197 & 184 & 145 & 116 & 58 & 22 & 10 & \text { IMRT } & 197 & 188 & 166 & 146 & 78 & 34 & 13\end{array}$

Figure 5. Clinical outcomes following IMRT and PBSPT after propensity score-adjusted analysis: LRC after PSM (A); OS after PSM (B); LRC after IPTW (C); OS after IPTW (D). Abbreviations: IMRT, intensity-modulated radiation therapy; PBSPT, pencil beam scanning proton therapy; LRC, locoregional control; OS, overall survival; PSM, propensity score matching; IPTW, inverse probability of treatment weighting. 
Table 6. Patient, tumor, and treatment characteristics after propensity score matching and weighting.

\begin{tabular}{ccccccc}
\hline (Reference: IMRT) & \multicolumn{3}{c}{ PSM } & & \multicolumn{2}{c}{ IPTW } \\
\hline & HR & $(95 \%$ CI $)$ & $p$-value & HR & $(95 \%$ CI) & $p$-value \\
\hline Locoregional control & 0.46 & $(0.13-1.67)$ & 0.236 & 0.87 & $(0.46-1.64)$ & 0.668 \\
Overall survival & 1.69 & $(0.54-5.29)$ & 0.371 & 1.39 & $(0.71-2.71)$ & 0.339 \\
\hline Acute esophagitis grade $\geq 3$ & OR & $(95 \%$ CI) & $p$-value & OR & $(95 \%$ CI) & $p$-value \\
Radiation pneumonitis grade $\geq 2$ & 2.91 & $(0.68-12.45)$ & 0.151 & 5.33 & $(1.21-23.46)$ & 0.028 \\
\hline
\end{tabular}

Abbreviations: IMRT, intensity modulated radiation therapy; PSM, propensity score matching; IPTW, inverse probability of treatment weighting; $\mathrm{HR}$, hazards ratio; $\mathrm{CI}$, confidence interval; OR, odds ratio.

\section{Discussion}

Given the recent advances of the scanning beam in PBT, the current results support the early clinical feasibility of PBSPT in definitive CCRT for NSCLC. PBSPT plans significantly reduced the radiation dose to the lung and spinal cord, with comparable target coverage. Showing comparable survival outcomes, PBSPT resulted in similar rates of symptomatic RP, even in patients who were relatively elderly and in those with poor pulmonary function, compared to those treated with IMRT. However, PBSPT was associated with frequent severe acute esophagitis, even with comparable dosimetric results.

Several plan comparison studies demonstrated that PBT with passive scattering could reduce the volume of lung, esophagus, and spinal cord by up to $30 \%$ compared to threedimensional conformal RT or even IMRT [8,9]. However, a recent randomized trial on PBT with passive scattering showed no benefit compared to IMRT in the doses to normal lung (mean, 16.1 vs. $16.6 \mathrm{~Gy})$, resulting in no significant difference in grade $\geq 3 \mathrm{RP}(10.5 \%$ vs. $6.5 \%$ ) [15]. A possible reason for these conflicting results might result from the technical issues associated with three-dimensional PBT with passive scattering. Recent planning studies of PBSPT showed significant improvements in sparing normal organs [17-20]. In the current study, both dosimetric results from the entire cohort and head-to-head plan comparison outcomes show consistent results of sparing dose to the normal lung, spinal cord, and heart. Further technical advancements in scanning performance and calculation algorithms could increase both the robustness and advantage in normal tissue sparing [16].

The incidence of grade $\geq 2(24.0 \%)$ or grade $\geq 3(8.0 \%)$ acute skin toxicities after PBSPT in the current study was relatively higher than historical data of $<5 \%$ regarding severe dermatitis (wet desquamation). A relatively high rate of grade $\geq 3$ dermatitis after PBT for treating NSCLC has been reported, ranging from $6 \%$ to $24 \%[10-12,14]$. Concerns remain regarding the increased dermatitis after PBT due to a higher entry dose of the spread-out Bragg peak of protons or the limited number of beams (2-3 per patient) to minimize the radiation dose to the normal lung $[14,27,28]$. Regarding dose constraints concerning skin, further cost function in planning PBSPT could reduce potential severe dermatitis [29]. The incidence of $20 \%$ for grade $\geq 3$ esophagitis seems comparable to that of the $18 \%$ obtained in the meta-analysis from a historical randomized trial of photon RT [4], while it appears to be more frequent than that of the $13.2 \%$ reported in the IMRT group from the secondary analysis of the RTOG 0617 trial [30]. A recent systemic review identified the dose-volume relationship in esophagitis regarding $\mathrm{V} 60 \mathrm{GyE}$, and the current study demonstrated that $\mathrm{V} 45 \mathrm{GyE} \geq 35 \%$ is associated with esophagitis [5]. However, further analysis adjusted for dose-volume parameters and IPTW analysis demonstrated that PBSPT could increase severe esophagitis despite the comparable dose distribution. We could speculate several possible reasons for frequent severe esophagitis, including robust optimization methods and RBE. Robust optimizations with additional margins to compensate for dose uncertainty could broaden distal falloff and stiffness of target coverage, resulting in unexpected exposure to the esophagus by the beam angle. Although a fixed value of 1.1 is commonly used as RBE for PBT, RBE itself shows various values according to depth, with the highest value observed near the distal edge of the beam [31]. 
However, further investigation regarding these technical and biological issues related to toxicities is required.

Since the incidence of grade $\geq 4 \mathrm{RP}$ after photon RT was relatively high, ranging from $18.2 \%$ to $35.7 \%$, in patients with poor lung function [32,33], physicians were forced to compromise CTV/PTV coverage or reduce the total dose to prevent severe lung toxicity in such patients [34]. In the current study, although the PBSPT group had poor baseline pulmonary function, there was no grade $\geq 3 \mathrm{RP}$ in the PBSPT group, and the pattern of changes in PFT was similar to that in the IMRT group. Since both mean lung dose and lung $\mathrm{V}_{5 \mathrm{GyE}-20 \mathrm{GyE}}$ have been reported to be associated with RP [6], PBSPT might influence the RP development. Despite an absence of long-term follow-up for cardiotoxicity in the current study, PBSPT could potentially reduce the radiation-induced cardiac toxic events resulting from reduced mean dose and $\mathrm{V}_{30 \mathrm{GyE}-50 \mathrm{GyE}}$ of the heart [6]. Atkins et al. suggested stringent avoidance of cardiac radiation dose based on an increased risk of cardiotoxicity and mortality with increasing cardiac dose in patients with locally advanced NSCLC [7]. The recent post hoc modeling study of the RTOG 0617 trial also showed a relationship between a higher dose to cardiopulmonary substructures and unexpected mortality [35]. The reduced dose to those structures of PBT might translate into improved survival of patients undergoing PBT compared to IMRT, which was observed in the National Cancer Database; this potential benefit could thus be maximized when adopting PBSPT [36]. Although there was no difference in major cardiac events between the PBSPT and IMRT groups in the current study, further follow-up of the current study and a more recent randomized trial would further validate the reduced incidence of cardiotoxicity after PBT and demonstrate a survival benefit.

There are some potential drawbacks in utilizing PBSPT. First, overall costs of PBT easily exceed those of photon RT, even after toxicity rate-adjusted analysis [37]. However, PBT increased the quality-adjusted life-years by 0.549 and 0.452 compared to 3D CRT/IMRT [38]. An ongoing RTOG 1308 trial (ClinicalTrials.gov: NCT01993810) will address cost effectiveness. Further cost-effectiveness analysis in patients with poor lung function should be considered. Second, although 31 centers are available for PBT in the United States [39], there is limited availability of PBT in other regions due to the higher cost of infrastructures relative to photon RT. Therefore, clear evidence demonstrating obvious clinical benefit is needed to justify the implementation of PBSPT in CCRT for NSCLC.

Although propensity score-adjusted analysis was undertaken, a major confounder cannot be adjusted due to the small sample size of the PBSPT group. Second, as a retrospective study, the physician-assessed toxicities should be interpreted with caution. However, our analysis was strengthened by use of PBSPT and by our inclusion of patients with poor pulmonary function. A recent randomized trial only included patients with FEV1 > 1.0 L; however, we observed $40 \%$ of patients in the PBSPT group with moderate-to-severe impaired pulmonary function. In addition, thorough individualized plan analysis not only for the entire patient cohort but also for the parallel patients in the PBSPT group could provide more detailed information. The relatively lower OS of the PBSPT group than the IMRT group might stem from a difference in age distribution; there was no difference in OS outcomes after PSM and IPTW analysis.

In conclusion, we note a possible benefit of PBSPT regarding tolerable toxicities with comparable survival outcomes based on real-world clinical data. Further randomized trials might be warranted to endorse PBSPT as an alternative treatment option in locally advanced NSCLC. 
Supplementary Materials: The following are available online at https: / www.mdpi.com/article/ 10.3390 / cancers 13143497 /s1, Figure S1: Baseline pulmonary function tests according to treatment modality: forced expiratory volume in $1 \mathrm{~s}$ (A); diffusing capacity of the lung for carbon monoxide (B), Figure S2: Changes in pulmonary function test after treatment: forced expiratory volume in $1 \mathrm{~s}(\mathrm{~A})$; diffusing capacity of the lung for carbon monoxide (B), Table S1: Dose volume parameters according to the treatment modality, Table S2: Comparison of target coverage and normal tissue sparing with matched intensity-modulated radiation therapy (IMRT) and intensity-modulated proton therapy (PBSPT) plans.

Author Contributions: Conceptualization: H.P.; Methodology: N.K., J.M.N. and H.P.; Formal analysis and investigation: All authors; Writing—original draft preparation: N.K., J.M.N.; Writing—review and editing: All authors; Funding acquisition: J.M.N., H.P.; Supervision: H.P. All authors have read and agreed to the published version of the manuscript.

Funding: This research was supported by a grant of the Korea Health Technology R\&D Project through the Korea Health Industry Development Institute (KHIDI), funded by the Ministry of Health and Welfare, Republic of Korea (grant number: HI19C0481, HC19C0293).

Institutional Review Board Statement: The study was conducted according to the guidelines of the Declaration of Helsinki, and approved by the Institutional Review Board of Samsung Medical Center (SMC 2020-01-034).

Informed Consent Statement: Patient consent was waived due to the retrospective nature of the current study.

Data Availability Statement: The datasets generated and analyzed during the current study are not publicly available due to institutional data protection law and confidentiality of patient data but are available from the corresponding author upon reasonable request in person.

Conflicts of Interest: The authors declare that the research was conducted in the absence of any commercial or financial relationships that could be construed as a potential conflict of interest.

\section{References}

1. Bradley, J.D.; Hu, C.; Komaki, R.R.; Masters, G.A.; Blumenschein, G.R.; Schild, S.E.; Bogart, J.A.; Forster, K.M.; Magliocco, A.M.; Kavadi, V.S.; et al. Long-term results of nrg oncology rtog 0617: Standard-versus high-dose chemoradiotherapy with or without cetuximab for unresectable stage iii non-small-cell lung cancer. J. Clin. Oncol. 2020, 38, 706-714. [CrossRef]

2. Machtay, M.; Bae, K.; Movsas, B.; Paulus, R.; Gore, E.M.; Komaki, R.; Albain, K.; Sause, W.T.; Curran, W.J. Higher biologically effective dose of radiotherapy is associated with improved outcomes for locally advanced non-small cell lung carcinoma treated with chemoradiation: An analysis of the radiation therapy oncology group. Int. J. Radiat. Oncol. Biol. Phys. 2012, 82, 425-434. [CrossRef]

3. De Ruysscher, D.; Faivre-Finn, C.; Nackaerts, K.; Jordan, K.; Arends, J.; Douillard, J.Y.; Ricardi, U.; Peters, S. Recommendation for supportive care in patients receiving concurrent chemotherapy and radiotherapy for lung cancer. Ann. Oncol. 2020, 31, 41-49. [CrossRef]

4. Aupérin, A.; Le Péchoux, C.; Rolland, E.; Curran, W.J.; Furuse, K.; Fournel, P.; Belderbos, J.; Clamon, G.; Ulutin, H.C.; Paulus, R.; et al. Meta-analysis of concomitant versus sequential radiochemotherapy in locally advanced non-small-cell lung cancer. J. Clin. Oncol. 2010, 28, 2181-2190. [CrossRef]

5. Palma, D.A.; Senan, S.; Oberije, C.; Belderbos, J.; de Dios, N.R.; Bradley, J.D.; Barriger, R.B.; Moreno-Jiménez, M.; Kim, T.H.; Ramella, S.; et al. Predicting esophagitis after chemoradiation therapy for non-small cell lung cancer: An individual patient data meta-analysis. Int. J. Radiat. Oncol. Biol. Phys. 2013, 87, 690-696. [CrossRef]

6. Simone, C.B., 2nd. Thoracic radiation normal tissue injury. Semin. Radiat. Oncol. 2017, 27, 370-377. [CrossRef]

7. Atkins, K.M.; Rawal, B.; Chaunzwa, T.L.; Lamba, N.; Bitterman, D.S.; Williams, C.L.; Kozono, D.E.; Baldini, E.H.; Chen, A.B.; Nguyen, P.L.; et al. Cardiac radiation dose, cardiac disease, and mortality in patients with lung cancer. J. Am. Coll. Cardiol. 2019, 73, 2976-2987. [CrossRef]

8. Chang, J.Y.; Zhang, X.; Wang, X.; Kang, Y.; Riley, B.; Bilton, S.; Mohan, R.; Komaki, R.; Cox, J.D. Significant reduction of normal tissue dose by proton radiotherapy compared with three-dimensional conformal or intensity-modulated radiation therapy in stage i or stage iii non-small-cell lung cancer. Int. J. Radiat. Oncol. Biol. Phys. 2006, 65, 1087-1096. [CrossRef] [PubMed]

9. Nichols, R.C.; Huh, S.N.; Henderson, R.H.; Mendenhall, N.P.; Flampouri, S.; Li, Z.; D'Agostino, H.J.; Cury, J.D.; Pham, D.C.; Hoppe, B.S. Proton radiation therapy offers reduced normal lung and bone marrow exposure for patients receiving dose-escalated radiation therapy for unresectable stage iii non-small-cell lung cancer: A dosimetric study. Clin. Lung Cancer 2011, 12, 252-257. [CrossRef] [PubMed] 
10. Oshiro, Y.; Okumura, T.; Kurishima, K.; Homma, S.; Mizumoto, M.; Ishikawa, H.; Onizuka, M.; Sakai, M.; Goto, Y.; Hizawa, N.; et al. High-dose concurrent chemo-proton therapy for stage iii nsclc: Preliminary results of a phase ii study. J. Radiat. Res. 2014, 55, 959-965. [CrossRef] [PubMed]

11. Chang, J.Y.; Verma, V.; Li, M.; Zhang, W.; Komaki, R.; Lu, C.; Allen, P.K.; Liao, Z.; Welsh, J.; Lin, S.H.; et al. Proton beam radiotherapy and concurrent chemotherapy for unresectable stage iii non-small cell lung cancer: Final results of a phase 2 study. JAMA Oncol. 2017, 3, e172032. [CrossRef]

12. Nguyen, Q.N.; Ly, N.B.; Komaki, R.; Levy, L.B.; Gomez, D.R.; Chang, J.Y.; Allen, P.K.; Mehran, R.J.; Lu, C.; Gillin, M.; et al. Long-term outcomes after proton therapy, with concurrent chemotherapy, for stage ii-iii inoperable non-small cell lung cancer. Radiother. Oncol. 2015, 115, 367-372. [CrossRef]

13. Hoppe, B.S.; Henderson, R.; Pham, D.; Cury, J.D.; Bajwa, A.; Morris, C.G.; D’Agostino, H., Jr.; Flampouri, S.; Huh, S.; Li, Z.; et al. A phase 2 trial of concurrent chemotherapy and proton therapy for stage iii non-small cell lung cancer: Results and reflections following early closure of a single-institution study. Int. J. Radiat. Oncol. Biol. Phys. 2016, 95, 517-522. [CrossRef]

14. Sejpal, S.; Komaki, R.; Tsao, A.; Chang, J.Y.; Liao, Z.; Wei, X.; Allen, P.K.; Lu, C.; Gillin, M.; Cox, J.D. Early findings on toxicity of proton beam therapy with concurrent chemotherapy for nonsmall cell lung cancer. Cancer 2011, 117, 3004-3013. [CrossRef]

15. Liao, Z.; Lee, J.J.; Komaki, R.; Gomez, D.R.; O’Reilly, M.S.; Fossella, F.V.; Blumenschein, G.R., Jr.; Heymach, J.V.; Vaporciyan, A.A.; Swisher, S.G.; et al. Bayesian adaptive randomization trial of passive scattering proton therapy and intensity-modulated photon radiotherapy for locally advanced non-small-cell lung cancer. J. Clin. Oncol. 2018, 36, 1813-1822. [CrossRef]

16. Han, Y. Current status of proton therapy techniques for lung cancer. Radiat. Oncol. J. 2019, 37, 232-248. [CrossRef]

17. Zhang, X.; Li, Y.; Pan, X.; Xiaoqiang, L.; Mohan, R.; Komaki, R.; Cox, J.D.; Chang, J.Y. Intensity-modulated proton therapy reduces the dose to normal tissue compared with intensity-modulated radiation therapy or passive scattering proton therapy and enables individualized radical radiotherapy for extensive stage iiib non-small-cell lung cancer: A virtual clinical study. Int. J. Radiat. Oncol. Biol. Phys. 2010, 77, 357-366. [PubMed]

18. Kesarwala, A.H.; Ko, C.J.; Ning, H.; Xanthopoulos, E.; Haglund, K.E.; O’Meara, W.P.; Simone, C.B., 2nd; Rengan, R. Intensitymodulated proton therapy for elective nodal irradiation and involved-field radiation in the definitive treatment of locally advanced non-small-cell lung cancer: A dosimetric study. Clin. Lung Cancer 2015, 16, 237-244. [CrossRef] [PubMed]

19. Stuschke, M.; Kaiser, A.; Pöttgen, C.; Lübcke, W.; Farr, J. Potentials of robust intensity modulated scanning proton plans for locally advanced lung cancer in comparison to intensity modulated photon plans. Radiother. Oncol. 2012, 104, 45-51. [CrossRef] [PubMed]

20. Chang, J.Y.; Li, H.; Zhu, X.R.; Liao, Z.; Zhao, L.; Liu, A.; Li, Y.; Sahoo, N.; Poenisch, F.; Gomez, D.R.; et al. Clinical implementation of intensity modulated proton therapy for thoracic malignancies. Int. J. Radiat. Oncol. Biol. Phys. 2014, 90, 809-818. [CrossRef] [PubMed]

21. Yu, N.Y.; DeWees, T.A.; Liu, C.; Daniels, T.B.; Ashman, J.B.; Beamer, S.E.; Jaroszewski, D.E.; Ross, H.J.; Paripati, H.R.; Rwigema, J.M.; et al. Early outcomes of patients with locally advanced non-small cell lung cancer treated with intensitymodulated proton therapy versus intensity-modulated radiation therapy: The mayo clinic experience. Adv. Radiat. Oncol. 2020, 5, 450-458. [CrossRef]

22. Choi, J.K.; Paek, D.; Lee, J.O. Normal predictive values of spirometry in korean population. Tuberc. Respir. Dis. 2005, 58, $230-242$. [CrossRef]

23. Pellegrino, R.; Viegi, G.; Brusasco, V.; Crapo, R.O.; Burgos, F.; Casaburi, R.; Coates, A.; van der Grinten, C.P.; Gustafsson, P.; Hankinson, J.; et al. Interpretative strategies for lung function tests. Eur. Respir. J. 2005, 26, 948-968. [CrossRef] [PubMed]

24. Yoo, G.S.; Yu, J.I.; Cho, S.; Jung, S.H.; Han, Y.; Park, S.; Oh, Y.; Lee, B.; Park, H.C.; Lim, D.H.; et al. Comparison of clinical outcomes between passive scattering versus pencil-beam scanning proton beam therapy for hepatocellular carcinoma. Radiother. Oncol. 2020, 146, 187-193. [CrossRef]

25. Fredriksson, A.; Forsgren, A.; Hårdemark, B. Minimax optimization for handling range and setup uncertainties in proton therapy. Med. Phys. 2011, 38, 1672-1684. [CrossRef] [PubMed]

26. Hicks, K.A.; Tcheng, J.E.; Bozkurt, B.; Chaitman, B.R.; Cutlio, D.E.; Farb, A.; Fonarow, G.C.; Jacobs, J.P.; Jaff, M.R.; Lichiman, J.H.; et al. 2014 ACC/AHA Key Data Elements and Definitions for Cardiovascular Endpoint Events in Clinical Trials: A Report of the American College of Cardiology/American Heart Association Task Force on Clinical Data Standards (Writing Committee to Develop Cardiovascular Endpoints Data Standards). J. Am. Coll. Cardiol. 2015, 66, 403-469. [PubMed]

27. Liang, X.; Bradley, J.A.; Zheng, D.; Rutenberg, M.; Yeung, D.; Mendenhall, N.; Li, Z. Prognostic factors of radiation dermatitis following passive-scattering proton therapy for breast cancer. Radiat. Oncol. 2018, 13, 72. [CrossRef]

28. DeCesaris, C.M.; Rice, S.R.; Bentzen, S.M.; Jatczak, J.; Mishra, M.V.; Nichols, E.M. Quantification of acute skin toxicities in patients with breast cancer undergoing adjuvant proton versus photon radiation therapy: A single institutional experience. Int. J. Radiat. Oncol. Biol. Phys. 2019, 104, 1084-1090. [CrossRef]

29. Tommasino, F.; Durante, M.; D’Avino, V.; Liuzzi, R.; Conson, M.; Farace, P.; Palma, G.; Schwarz, M.; Cella, L.; Pacelli, R. Model-based approach for quantitative estimates of skin, heart, and lung toxicity risk for left-side photon and proton irradiation after breast-conserving surgery. Acta Oncol. 2017, 56, 730-736. [CrossRef]

30. Chun, S.G.; Hu, C.; Choy, H.; Komaki, R.U.; Timmerman, R.D.; Schild, S.E.; Bogart, J.A.; Dobelbower, M.C.; Bosch, W.; Galvin, J.M.; et al. Impact of intensity-modulated radiation therapy technique for locally advanced non-small-cell lung cancer: A secondary analysis of the nrg oncology rtog 0617 randomized clinical trial. J. Clin. Oncol. 2017, 35, 56-62. [CrossRef] [PubMed] 
31. Saager, M.; Peschke, P.; Brons, S.; Debus, J.; Karger, C.P. Determination of the proton rbe in the rat spinal cord: Is there an increase towards the end of the spread-out bragg peak? Radiother. Oncol. 2018, 128, 115-120. [CrossRef]

32. Kim, H.; Pyo, H.; Noh, J.M.; Lee, W.; Park, B.; Park, H.Y.; Yoo, H. Preliminary result of definitive radiotherapy in patients with non-small cell lung cancer who have underlying idiopathic pulmonary fibrosis: Comparison between x-ray and proton therapy. Radiat. Oncol. 2019, 14, 19. [CrossRef]

33. Lee, Y.H.; Kim, Y.S.; Lee, S.N.; Lee, H.C.; Oh, S.J.; Kim, S.J.; Kim, Y.K.; Han, D.H.; Yoo Ie, R.; Kang, J.H.; et al. Interstitial lung change in pre-radiation therapy computed tomography is a risk factor for severe radiation pneumonitis. Cancer Res. Treat. 2015, 47, 676-686. [CrossRef] [PubMed]

34. Kim, H.; Yoo, H.; Pyo, H.; Ahn, Y.C.; Noh, J.M.; Ju, S.G.; Lee, W.; Park, B.; Kim, J.M.; Kang, N.; et al. Impact of underlying pulmonary diseases on treatment outcomes in early-stage non-small cell lung cancer treated with definitive radiotherapy. Int. J. Chron. Obstruct. Pulmon. Dis. 2019, 14, 2273-2281. [CrossRef] [PubMed]

35. Thor, M.; Deasy, J.O.; Hu, C.; Gore, E.; Bar-Ad, V.; Robinson, C.; Wheatley, M.; Oh, J.H.; Bogart, J.; Garces, Y.I.; et al. Modeling the impact of cardio-pulmonary irradiation on overall survival in nrg oncology trial rtog 0617. Clin. Cancer Res. 2020. [CrossRef]

36. Higgins, K.A.; O'Connell, K.; Liu, Y.; Gillespie, T.W.; McDonald, M.W.; Pillai, R.N.; Patel, K.R.; Patel, P.R.; Robinson, C.G.; Simone, C.B., 2nd; et al. National cancer database analysis of proton versus photon radiation therapy in non-small cell lung cancer. Int. J. Radiat. Oncol. Biol. Phys. 2017, 97, 128-137. [CrossRef]

37. Smith, W.P.; Richard, P.J.; Zeng, J.; Apisarnthanarax, S.; Rengan, R.; Phillips, M.H. Decision analytic modeling for the economic analysis of proton radiotherapy for non-small cell lung cancer. Transl. Lung Cancer Res. 2018, 7, 122-133. [CrossRef] [PubMed]

38. Lievens, Y.; Verhaeghe, N.; Neve, W.D.; Madani, I.; Vanderstraeten, B.; Verstraete, J.; Annemans, L. Proton radiotherapy for locally-advanced non-small cell lung cancer, a cost-effective alternative to photon radiotherapy in belgium? J. Thorac. Oncol. 2013, 8, S839-S840.

39. Particle Therapy Co-Operative Group. Particle Therapy Patient Statistics (per end of 2018). Available online: https://www.ptcog. ch/images/patientstatistics/Patientstatistics-updateDec2018.pdf (accessed on 22 May 2020). 